\title{
Local Inversion Of The Sonar Transform Regularized By The Approximate Inverse
}

\author{
E.T. Quinto \\ A. Rieder \\ T. Schuster \\ Preprint Nr. 10/07
}

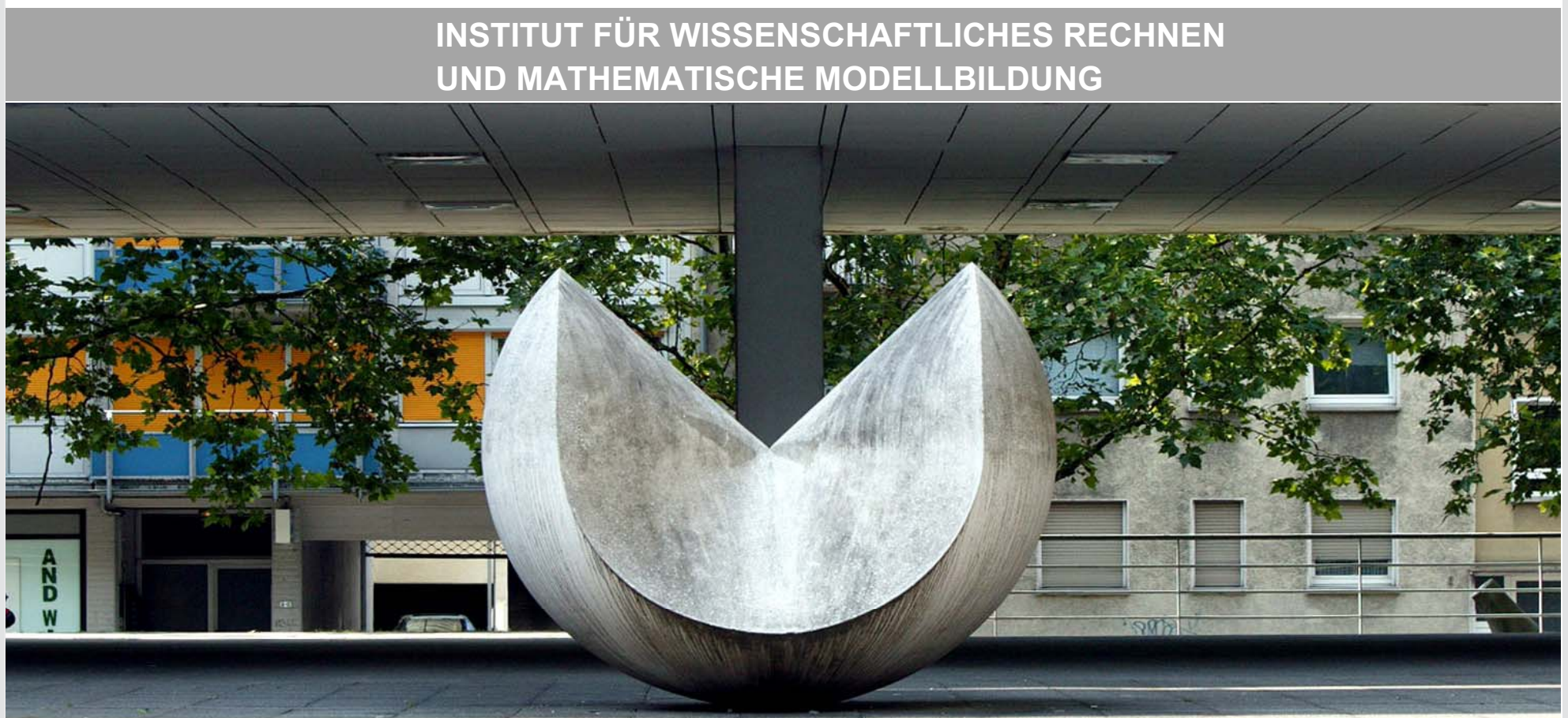

KIT - Universität des Landes Baden-Württemberg und 


\section{Anschriften der Verfasser:}

Prof. Dr. Eric Todd Quinto

Department of Mathematics

Tufts University

Medford MA 02155 USA

Prof. Dr. Andreas Rieder

Institut für Angewandte und Numerische Mathematik

Karlsruher Institut für Technologie (KIT)

D-76128 Karlsruhe

Prof. Dr. Thomas Schuster

Fakultät V, Institut für Mathematik

Carl von Ossietzky Universität Oldenburg

D-26111 Oldenburg 


\title{
LOCAL INVERSION OF THE SONAR TRANSFORM REGULARIZED BY THE APPROXIMATE INVERSE
}

\author{
ERIC TODD QUINTO, ANDREAS RIEDER, AND THOMAS SCHUSTER
}

\begin{abstract}
A new reconstruction method is given for the spherical mean transform with centers on a plane in $\mathbb{R}^{3}$ which is also called Sonar transform. Standard inversion formulas require data over all spheres, but typically, the data are limited in the sense that the centers and radii are in a compact set. Our reconstruction operator is local because, to reconstruct at $\mathbf{x}$, one needs only spheres that pass near $\mathbf{x}$, and the operator reconstructs singularities, such as object boundaries. The microlocal properties of the reconstruction operator, including its symbol as a pseudodifferential operator, are given. The method is implemented using the approximate inverse, and reconstructions are given. They are evaluated in light of the microlocal properties of the reconstruction operator.
\end{abstract}

A version of this preprint containing color figures can be downloaded under www.math.kit.edu/ianm3/ rieder/media/local_sonar.pdf.

\section{Introduction}

In this article, we develop a novel local reconstruction method for the spherical Radon transform with centers on a plane. As this transform is one model for Sonar under the Born approximation, that is, under the assumption there are not multiple scattering events, it is also called Sonar transform.

Let $u(t ; \mathbf{x})$ be the acoustic pressure field at $\mathbf{x} \in \mathbb{R}^{3}$ at time $t \geq 0$. Then, $u$ satisfies the acoustic wave equation

$$
\Delta_{\mathbf{x}} u-\frac{1}{\nu^{2}} \partial_{t}^{2} u=-\delta(\mathbf{x}-\mathbf{z}) \delta(t)
$$

where $\nu=\nu(\mathbf{x})$ is the speed of sound and $\mathbf{z} \in \mathcal{P}=\left\{\mathbf{x} \in \mathbb{R}^{3} \mid x_{3}=0\right\}$ is the excitation point on the ocean surface. The inverse problem in Sonar is to recover $\nu$ from the backscattered (reflected) field $u_{\mathrm{s}}$ observed at $\mathcal{P}$ for all times $t>0$.

Cohen and Bleistein [3] made the ansatz

$$
\frac{1}{\nu^{2}(\mathbf{x})}=\frac{1+n(\mathbf{x})}{c^{2}}
$$

where $c$ is a constant background velocity. Then,

$$
\frac{1}{4 \pi} \frac{1}{c^{2} \tau^{2}} \int_{\mathcal{S}\left(\mathbf{y}, \frac{c \tau}{2}\right)} n(\mathbf{x}) d S(\mathbf{x})=-c^{2} \int_{0}^{\tau}(\tau-t) u_{\mathbf{s}}(t, \mathbf{y}) d t+\text { higher order terms in } n
$$

Date: September 27, 2010.

1991 Mathematics Subject Classification. Primary: 44A12, 65R20.

The first author was supported in part by NSF Grant DMS 0908015 (and DMS 0456858 for preliminary work) and the Alexander von Humboldt Stiftung. He thanks Karlsruher Institut für Technologie (formerly Universität Karlsruhe) for its hospitality while this work was begun. 
where $\mathcal{S}(\mathbf{y}, r)$ is the sphere centered at $\mathbf{y} \in \mathbb{R}^{3}$ and of radius $r$ and $\tau$ is the observation period. Under the assumption that $n \ll 1$ (i.e. the Born approximation), the higher order terms are set to zero and the right-hand side of (1.2) becomes an integral from 0 to $\tau$ of the solution to the wave equation. Thus, (1.2) reduces to recovering $n(\mathbf{x})$ from integrals of $n$ over spheres centered on the plane $\mathcal{P}$ where the right had side in (1.2) is known from the measured data $u(t, \mathbf{y})$.

Since we are interested in spheres with centers on the plane $x_{3}=0$, we define our spheres in terms of $\mathbf{z} \in \mathbb{R}^{2}$ and $r>0$

$$
S(\mathbf{z}, r)=\left\{\mathbf{x} \in \mathbb{R}^{3}|| \mathbf{x}-(\mathbf{z}, 0) \mid=r\right\}, \quad Y=\left\{(\mathbf{z}, r) \mid \mathbf{z} \in \mathbb{R}^{2}, r>0\right\} .
$$

We define the spherical Radon transform for $(\mathbf{z}, r) \in Y$ to be the spherical mean over $S(\mathbf{z}, r)$ :

$$
R f(\mathbf{z}, r)=\frac{1}{4 \pi r^{2}} \int_{S(\mathbf{z}, r)} n(\mathbf{x}) d S(\mathbf{x}) .
$$

Our goal is to use this spherical mean data to reconstruct a picture of $f$ showing region boundaries. Since the null space of $R$ is the set of odd functions [4], $R$ is not injective for arbitrary functions on $\mathbb{R}^{3}$. Courant and Hilbert's null space characterization implies $R$ is injective for functions supported in $x_{3}>0$. We let

$$
\mathbb{R}_{+}^{3}=\left\{\mathbf{x} \in \mathbb{R}^{3} \mid x_{3}>0\right\}
$$

and we will consider only functions supported in $\mathbb{R}_{+}^{3}$. This is a realistic assumption for functions in the ocean when we assume $x_{3}>0$ points down to the ocean floor.

We define the backprojection operator $R^{*}$ for compactly supported functions $g(\mathbf{z}, r)$ as

$$
R^{*} g(\mathbf{x})=\int_{\mathbb{R}^{2}} g(\mathbf{z},|\mathbf{x}-(\mathbf{z}, 0)|) d \mathbf{z} .
$$

Note that

$$
|\mathbf{x}-(\mathbf{z}, 0)|=\sqrt{\left|\mathbf{x}^{\prime}-\mathbf{z}\right|^{2}+x_{3}^{2}} \text { where } \mathbf{x}^{\prime}=\left(x_{1}, x_{2}, x_{3}\right)^{\prime}:=\left(x_{1}, x_{2}\right) .
$$

The operator $R^{*}$ is used in $[2,6]$ and it is the dual operator to $R$ if the measure on $\mathbb{R}_{+}^{3}$ is $d x$ and the measure on $Y$ is $4 \pi r^{2} d r d \mathbf{z}$. The problem is that one cannot define $R^{*}$ on the range of $R$ even for compactly supported functions $f$ because $R f$ is not necessarily compactly supported even if $f$ is. Therefore we will need to include a cutoff function, see (2.2) below, in the definition of our reconstruction operator.

Inversion algorithms for this problem exist if data are known over all spheres with center on a plane $[17,2,6,13]$. For the two-dimensional problem, Palamodov [18] analyzed the visible and invisible singularities, providing seminorm strength estimates for each, and Denisjuk [5] developed inversion algorithms. Schuster and Quinto [25] adapted the approximate inverse to distributions and used it to develop an inversion algorithm for the two-dimensional problem. This model, integration over spheres, also comes up in thermoacoustic and photoacoustic tomography, but in this case the centers are constrained to lie on a sphere or other surface that encloses the region to be imaged $([1,11,14,27]$ provide references and background).

Our reconstruction operator is local in the sense that to reconstruct at a point $\mathbf{x}$, one needs only spheres that are near $\mathbf{x}$, and the operator is easily restricted to the data that are given in practice. Typical data are limited since one can acquire data only over a compact set in $Y$, and the authors know of no reconstruction method from this limited data in $\mathbb{R}^{3}$. Our reconstruction operator will detect singularities such as boundaries of the object rather than finding reflectivity values, and as shown in Sect. 5, the operator can image objects clearly. Furthermore, our algorithm is easy 
to adapt to different data acquisition geometries, such as when $\mathbf{z}$ lies on an arbitrary $C^{\infty}$ surface rather than a plane.

In Section 2 we define our reconstruction operator and give its basic properties. To understand why and how our algorithm detects singularities we will analyze its (principal) symbol as a pseudodifferential operator ( $\Psi D O)$ (Section 3). In Section 4 we use the approximate inverse to regularize our inversion operator. To this end we analytically compute a reconstruction kernel from a given mollifier (Theorem 4.1). Finally, we present several fully 3D numerical experiments in Section 5 and analyze the resulting reconstructions in the light of the microlocal results we developed in Section 3. The technical proof of Theorem 4.1 is given in the appendix.

\section{Our local Reconstruction Operator}

In contrast to the planar Radon transform that integrates over planes, the spherical Radon transform $R$ cannot be formulated as bounded operator neither between appropriate $L^{2}$-, nor Sobolev spaces. Moreover Andersson [2] proves that $R$ acts as a bounded mapping between suitable chosen spaces of tempered distributions. Let

$$
\mathcal{S}_{\mathrm{e}}\left(\mathbb{R}^{3}\right):=\left\{f \in \mathcal{S}\left(\mathbb{R}^{3}\right) \mid f\left(\mathbf{x}^{\prime},-x_{3}\right)=f\left(\mathbf{x}^{\prime}, x_{3}\right)\right\}
$$

be the space of rapidly decreasing functions that are even in $x_{3}$ and let

$$
\mathcal{S}_{\mathrm{r}}\left(\mathbb{R}^{2} \times \mathbb{R}^{3}\right)=\left\{f \in \mathcal{S}\left(\mathbb{R}^{5}\right) \mid f(\mathbf{z}, w)=\check{f}(z,\|w\|) \text { for a function } \check{f} \in \mathcal{S}_{\mathrm{e}}\left(\mathbb{R}^{3}\right)\right\}
$$

be the space of rapidly decreasing functions in $\mathbb{R}^{5}$ that are radially symmetric in the last three components. The dual spaces $\mathcal{S}_{\mathrm{e}}\left(\mathbb{R}^{3}\right)^{\prime}$ and $\mathcal{S}_{\mathrm{r}}\left(\mathbb{R}^{2} \times \mathbb{R}^{3}\right)^{\prime}$ of $\mathcal{S}_{\mathrm{e}}\left(\mathbb{R}^{3}\right)$ and $\mathcal{S}_{\mathrm{r}}\left(\mathbb{R}^{2} \times \mathbb{R}^{3}\right)$, respectively, consist of tempered distributions. In general $f \in \mathcal{S}_{\mathrm{e}}\left(\mathbb{R}^{3}\right)$ does not imply that $R f \in \mathcal{S}_{\mathrm{r}}\left(\mathbb{R}^{2} \times \mathbb{R}^{3}\right)$, but it is easy to show that $R f \in \mathcal{S}_{\mathrm{r}}\left(\mathbb{R}^{2} \times \mathbb{R}^{3}\right)^{\prime}$. By a density argument we derive that $R$ maps $\mathcal{S}_{\mathrm{e}}\left(\mathbb{R}^{3}\right)^{\prime}$ to $\mathcal{S}_{\mathrm{r}}\left(\mathbb{R}^{2} \times \mathbb{R}^{3}\right)^{\prime}$ and Andersson [2, Th. 2.1] proved that this gives a bounded operator whose adjoint maps $\mathcal{S}_{\mathrm{r}}\left(\mathbb{R}^{2} \times \mathbb{R}^{3}\right)$ to $\mathcal{S}_{\mathrm{e}}\left(\mathbb{R}^{3}\right)$,

$$
R^{*}: \mathcal{S}_{\mathrm{r}}\left(\mathbb{R}^{2} \times \mathbb{R}^{3}\right) \rightarrow \mathcal{S}_{\mathrm{e}}\left(\mathbb{R}^{3}\right)
$$

and has dense range. As a consequence we see that the composition $R^{*} R$ is not meaningfully defined in general and this is the reason for introducing a cutoff function $\phi$ in (2.2) below to obtain $\phi R f \in \mathcal{S}_{\mathrm{r}}\left(\mathbb{R}^{2} \times \mathbb{R}^{3}\right)$.

We use the following notation. Let $\Delta$ be the full Laplacian in $\mathbb{R}^{3}$. We let $H_{x_{3}}$ be the Hilbert transform in $x_{3}$ (the Fourier multiplier with symbol $-i \operatorname{sgn}\left(\xi_{3}\right)[26]$ ), and we let $\partial_{x_{3}}=\partial / \partial x_{3}$.

Our local algorithm starts from an exact formula of Klein [13] that is based on work of Andersson [2] and Fawcett [6]. The formula of Klein involves a modified dual operator that includes derivatives with respect to $\mathbf{x}$ of the data. He proves that the addition of the derivative allows one to compose the dual operator with $R$ for functions in the range of $R$ [13]. Klein's formula in $\mathbb{R}^{3}$ is

$$
f=\frac{1}{2 \pi} H_{x_{3}} \Delta^{1 / 2} \int_{\mathbb{R}^{2}}\left(\left.\partial_{x_{3}} R f(\mathbf{z}, r)\right|_{r=\sqrt{\left|\mathbf{x}^{\prime}-\mathbf{z}\right|^{2}+x_{3}^{2}}}\right) d \mathbf{z} .
$$

The integral in (2.1) is the $R^{*}$ integral but with a $\partial_{x_{3}}$ inside the integral.

Now we make (2.1) local. We replace the non-local operator $\Delta^{1 / 2}$ by $\Delta$, and we replace the Hilbert transform $H_{x_{3}}$ (a pseudodifferential operator of order zero) by -1 . We use -1 rather than 1 so that the symbol of our operator $\Lambda$ (3.2) is positive for $\xi_{3}>0$. The replacement of $\sqrt{\Delta}$ by $\Delta$ increases the order of the operator from order zero (the order of the identity) to order one. 
For convenience, we replace the constant in our formula $\frac{1}{2 \pi}$ by 1 . Finally, we specify constants $0<T^{\prime}<T$ and $0<\delta<\delta^{\prime}<M^{\prime}<M$ and choose a $C^{\infty}$ cutoff in $\mathbf{z}$ and in $r$

$$
\begin{gathered}
\phi: \mathbb{R}^{2} \times(0, \infty) \rightarrow[0,1], \operatorname{supp}(\phi)=[-T, T]^{2} \times[\delta, M], \\
\phi(\mathbf{z}, r)>0 \quad(\mathbf{z}, r) \in(-T, T)^{2} \times(\delta, M), \\
\phi(\mathbf{z}, r)=1 \quad(\mathbf{z}, r) \in\left[-T^{\prime}, T^{\prime}\right]^{2} \times\left[\delta^{\prime}, M^{\prime}\right] .
\end{gathered}
$$

Including $\phi$ allows us to compose $R^{*}$ and $R$ even when the data $R f$ is not compactly supported. Multiplying by $\phi(\mathbf{z}, r)$ before using $\partial_{x_{3}}$ allows us to bring the derivative $\partial_{x_{3}}$ outside the $R^{*}$ integral to get our reconstruction operator for $\mathbf{x} \in \mathbb{R}^{3}$ :

$$
\Lambda f(\mathbf{x}):=-\Delta \partial_{x_{3}} R^{*}(\phi(\mathbf{z}, r) R f(\mathbf{z}, r))(\mathbf{x}) .
$$

This operator is a natural generalization of the Lambda tomography operator [7] since it is of order one as we will claim in Theorem 3.2 and it is local in the following sense. To reconstruct $\Lambda f(\mathbf{x})$ one needs only spheres near $\mathbf{x}$ to calculate the derivatives and to evaluate $R^{*}$.

\section{The microlocal Properties of $\Lambda$ And its Symbol as a $\Psi$ DO}

In this section, we give the microlocal properties of $R$. We prove $\Lambda$ is an elliptic $\Psi D O$ on $\mathbb{R}_{+}^{3}$ and we give its symbol. This will show how much $\Lambda$ emphasizes singularities in different directions. In order to understand what $R$ and $\Lambda$ do to singularities, we must first understand what singularities are and this will be framed in terms of the wavefront set.

For $f \in L^{1}\left(\mathbb{R}^{n}\right)$ we define the Fourier transform of $f$ to be

$$
\mathcal{F} f(\xi)=\frac{1}{(2 \pi)^{n / 2}} \int_{\mathbb{R}^{n}} f(\mathbf{x}) \exp (-i \xi \cdot \mathbf{x}) d \mathbf{x}
$$

and we note that $\mathcal{F}^{-1} f(\mathbf{x})=\mathcal{F} f(-\mathbf{x})$. Therefore, if $\mathcal{F} f$ is rapidly decreasing at $\infty$ (decreasing faster than any power of $1 /|\xi|$ at $\infty$ ) then $f$ and all its derivatives are continuous; that is $f \in C^{\infty}\left(\mathbb{R}^{n}\right)$. This is the motivation for the definition of wavefront set: we can understand smoothness of $f$ by understanding where $\mathcal{F} f$ is rapidly decreasing. We note that a cutoff function at $\mathbf{x}_{0}$ will be any $C^{\infty}$ compactly supported function $\varphi: \mathbb{R}^{n} \rightarrow[0, \infty)$ such that $\varphi\left(\mathbf{x}_{0}\right) \neq 0$. This motivates our next definition.

Definition 3.1. Let $f$ be a distribution in $\mathbb{R}^{n}$ and let $\mathbf{x}_{0} \in \mathbb{R}^{n}$ and $\xi_{0} \in \mathbb{R}^{n} \backslash \mathbf{0}$. Then $f$ is smooth at $\mathbf{x}_{0}$ in direction $\xi_{0}$ if for some cutoff function $\varphi$ at $\mathbf{x}_{0}$ and some open conic neighborhood $V$ of $\xi_{0}, \mathcal{F} f$ is rapidly decreasing for $\xi \in V$.

If $f$ is not smooth at $\mathbf{x}_{0}$ in direction $\xi_{0}$ then we say $\left(\mathbf{x}_{0}, \xi_{0}\right) \in \mathrm{WF}(f)$.

Using our next definition, we can evaluate qualitative strength of singularities.

Definition 3.2 ([19]). Let $f$ be a distribution in $\mathbb{R}^{n}$ and let $\mathbf{x}_{0} \in \mathbb{R}^{n}$ and $\xi_{0} \in \mathbb{R}^{n} \backslash \mathbf{0}$. Then $f$ is in $H^{s}$ at $\mathbf{x}_{0}$ in direction $\xi_{0}$ if for some cutoff function $\varphi$ at $\mathbf{x}_{0}$ and some open conic neighborhood $V$ of $\xi_{0}$, the microlocal Sobolov seminorm

is finite.

$$
\|\varphi f\|_{H^{s}, V}=\sqrt{\int_{V}|\mathcal{F}(\varphi f)(\xi)|^{2}\left(1+|\xi|^{2}\right)^{s} d \xi}
$$

If $f$ is not in $H^{s}$ at $\mathbf{x}_{0}$ in direction $\xi_{0}$ then we say $\left(\mathbf{x}_{0}, \xi_{0}\right) \in \mathrm{WF}^{\mathrm{s}}(f)$. 
Since $Y$ can be viewed as an open subset of $\mathbb{R}^{3}$, one can define $\mathrm{WF}$ and $\mathrm{WF}^{\mathrm{s}}$ for distributions on $Y$ because when one multiplies by a cutoff $\varphi(\mathbf{z}, r)$ that is compactly supported in $Y$, the $\mathbb{R}^{3}$ definition of Fourier transform can be used. In general, the wavefront set and Sobolev wavefront sets are defined as subsets of the cotangent bundle, $T^{*}\left(\mathbb{R}^{n}\right)$ because this allows one to define the wavefront set invariantly on manifolds.

Note that if $f$ is smooth on $\mathbb{R}^{n}$ then $\operatorname{WF}(f)=\emptyset$. If $f$ is the characteristic function $\chi_{\Omega}$ of a domain $\Omega$ with $C^{\infty}$ boundary then $\operatorname{WF}\left(\chi_{\Omega}\right)$ is the normal bundle of the boundary

$$
N(\operatorname{bd}(\Omega))=\left\{(\mathbf{x}, \xi) \mid \mathbf{x} \in \operatorname{bd}(\Omega), \xi \in \mathbb{R}^{3} \backslash \mathbf{0}, \xi \perp \operatorname{bd}(\Omega) \text { at } \mathbf{x}\right\}=\operatorname{WF}\left(\chi_{\Omega}\right) .
$$

This is also true for $\mathrm{WF}^{\mathrm{s}}(f)$ for $s \geq 1 / 2$.

Radon transforms detect singularities perpendicular to the set being integrated over and so $R$ will detect singularities of $f$ normal to the sphere being integrated over. This is made clear in the following theorem, a more precise version was given in [16] and was proven for manifolds in arbitrary dimensions in [21].

Theorem 3.1. Let $R$ be the spherical Radon transform in $\mathbb{R}^{3}$ with centers on the plane $x_{3}=0$. Then, $R$ is an elliptic Fourier integral operator [12] for functions supported in $\mathbb{R}_{+}^{3}$. Let $f$ be a locally integrable function on $\mathbb{R}_{+}^{3}$ and $\mathbf{z} \in \mathbb{R}^{2}$ and $r>0$. Rf is $C^{\infty}$ near $(\mathbf{z}, r)$ if and only if $\mathrm{WF}(f) \cap N(S(\mathbf{z}, r))=\emptyset$.

In [21] a precise relationship is given between $\mathrm{WF}(f)$ and $\mathrm{WF}(R f)$ but the simple version given here explains that $R$ "sees" singularities only if they are normal to the sphere in question.

Example 3.1. To illustrate our theorem, we give a basic example. Let $f$ be the characteristic function of a domain $\Omega \subset \mathbb{R}_{+}^{3}$ with $C^{\infty}$ boundary. According to Theorem 3.1, a singularity of $f$ will be visible in $R f$ near $(\mathbf{z}, r)$ if and only if the sphere $S(\mathbf{z}, r)$ is tangent to $\operatorname{bd}(\Omega)$ (so normals to the boundary are normal to the sphere). Our reconstructions in section 5 are from a limited set of spheres, and the only singularities that are visible in those reconstructions are the ones normal to spheres in the data set. Palamodov referred to the wavefront directions normal to $S(\mathbf{z}, r)$ as audible, and he proved elegant estimates for singularities in the audible zone (and inaudible zone) for the circle transform in $\mathbb{R}^{2}[18]$.

Now that we have discussed the microlocal properties of $R$, we consider $\Lambda$. We first prove that $\Lambda$ is a $\Psi D O$ and we give its symbol and then we discuss what the symbol means for the algorithm.

Theorem 3.2. Let $\Lambda$ be the operator (2.3) with $C^{\infty}$ cutoff function $\phi(2.2)$. Then, $\Lambda$ is a pseudodifferential operator of order one on $\mathcal{E}^{\prime}\left(\mathbb{R}_{+}^{3}\right)$. Furthermore the symbol of $\Lambda$ is

$$
\sigma(\Lambda)=2 \pi \phi\left(\left(\mathbf{x}-\frac{x_{3}}{\xi_{3}} \xi\right)^{\prime}, \frac{x_{3}}{\left|\xi_{3}\right|}|\xi|\right) \frac{\xi_{3}}{\left|\xi_{3}\right|}|\xi| .
$$

Before we prove the theorem we make some observations about what this means for our problem.

Note that our domain is $\mathbb{R}_{+}^{3}$ and so $x_{3}$ is always positive. The operator $\Lambda$ is not elliptic since $\sigma(\Lambda)$ can be zero as $\phi$ can be zero. For $\mathbf{x} \in \mathbb{R}_{+}^{3}$, let

$$
C(\mathbf{x})=\left\{\xi \in \mathbb{R}^{3}\left|\xi_{3} \neq 0,\left(\mathbf{x}-\frac{x_{3}}{\xi_{3}} \xi\right)^{\prime} \in(-T, T)^{2}, x_{3}\right| \xi|/| \xi_{3} \mid \in(\delta, M)\right\} .
$$

The symbol of $\Lambda$ is zero off of $\mathrm{Cl}(C(\mathbf{x}))$. Where the symbol is zero tells where the operator $\Lambda$ smooths, so $\Lambda f$ will not show any wavefront of $f$ at $(\mathbf{x}, \xi)$ if $\xi \notin C(\mathbf{x})$ (see Remark 3.3 below). 
Since the symbol $\sigma(\Lambda)$ is nonzero on $C(\mathbf{x})$ and homogeneous of degree one in $\xi$, if $\xi \in C(\mathbf{x})$, then $\Lambda$ is elliptic of order one at $(\mathbf{x}, \xi)[19]$. Therefore, if $\xi \in C(\mathbf{x})$, then $(\mathbf{x}, \xi) \in \mathrm{WF}(\Lambda f)$ if and only if $(\mathbf{x}, \xi) \in \mathrm{WF}(f)$. Thus wavefront of $f$ for $\xi \in C(\mathbf{x})$ will, in some sense, be visible in $\Lambda f$. Since $\Lambda$ has degree one and is elliptic on $C(\mathbf{x})$, singularities of $\Lambda f$ will be one degree less smooth in Sobolev scale than those of $f$. Of course wavefront directions near bd $(C(\mathbf{x}))$ might be reconstructed more weakly than those corresponding to where $\phi=1$. This discussion proves the following corollary.

Corollary 3.1. Let $\Lambda$ be the operator (2.3) with $C^{\infty}$ cutoff function $\phi(2.2)$. Let $\mathbf{x} \in \mathbb{R}_{+}^{3}$ and let $\xi \in C(\mathbf{x})$. Then,

$$
(\mathbf{x}, \xi) \in \mathrm{WF}^{\mathrm{s}}(f) \text { if and only if }(\mathbf{x}, \xi) \in \mathrm{WF}^{s-1}(\Lambda f) \text {. }
$$

Finally, we should emphasize that Theorem 3.2 and Corollary 3.1 are valid because we are considering only functions supported on one side of the plane $x_{3}=0$. Since the null space of $R$ is the set of odd functions about $x_{3}=0, R$ cannot distinguish singularities above $\left(\mathbf{x}^{\prime}, x_{3}\right)$ from those above $\left(\mathbf{x}^{\prime},-x_{3}\right)$. However, for functions supported in $\mathbb{R}_{+}^{3}$, this is not a problem.

Proof of Theorem 3.2. We will give an easy to understand explanation of the result and then we outline the proof.

For purposes of this heuristic discussion, we assume we can compose $R^{*}$ and $R$ and write $R^{*} \partial_{x_{3}}=$ $\partial_{x_{3}} R^{*}$ without having the cutoff $\phi$. These assumptions are wrong in general, but this calculation shows what result we should expect, and it will allow us to skip one step in the rigorous calculation. By Klein's formula, $I d=\frac{1}{2 \pi} H_{x_{3}} \Delta^{1 / 2} \partial_{x_{3}} R^{*} R$, and the symbol of $I d$ is 1 . Recall that $\sigma\left(H_{x_{3}}\right)=$ $-i \operatorname{sgn}\left(\xi_{3}\right)=-i \xi_{3} /\left|\xi_{3}\right|$ and $H_{x_{3}}^{-1}=-H_{x_{3}}$ [26]. Our operator $\Lambda$ without the cutoff $\phi$ is then $-2 \pi\left(-H_{x_{3}}\right) \sqrt{\Delta} I d$ and therefore the symbol of $\Lambda$ without the $\phi$ is $-2 \pi(i) \frac{\xi_{3}}{\left|\xi_{3}\right|}(i)|\xi|=2 \pi \frac{\xi_{3}}{\left|\xi_{3}\right|}|\xi|$.

To prove the theorem rigorously, we add the cutoff $\phi$ and we calculate the symbol of the composition of the Fourier integral operators that make up $\Lambda$. This starts with the canonical relation of $R$. For this proof, we will use cotangent notation. For $\xi=\left(\xi_{1}, \xi_{2}, \xi_{3}\right)$ we denote $\xi \mathbf{d x}=\xi_{1} \mathbf{d} \mathbf{x}_{1}+\xi_{2} \mathbf{d} \mathbf{x}_{2}+\xi_{3} \mathbf{d} \mathbf{x}_{3}$ and $\left(\eta_{1}, \eta_{2}\right) \mathbf{d z}=\eta_{1} \mathbf{d} \mathbf{z}_{1}+\eta_{2} \mathbf{d} \mathbf{z}_{2}$. Then the canonical relation of $R$ is $[21]^{1}$

$$
\mathcal{C}=\left\{\left(\mathbf{z}, r, \mathbf{x} ; \alpha\left(\left(\mathbf{x}^{\prime}-\mathbf{z}\right) \mathbf{d} \mathbf{z}+r \mathbf{d} \mathbf{r}-(\mathbf{x}-(\mathbf{z}, 0)) \mathbf{d} \mathbf{x}\right)\right) \mid \mathbf{x} \in \mathbb{R}_{+}^{3},(\mathbf{z}, r) \in Y, \alpha \neq 0\right\} .
$$

Since $\mathbf{x} \in \mathbb{R}_{+}^{3}$ we can specify global coordinates on $\mathcal{C}$ where we let $S_{+}^{2}=\left\{\omega \in S^{2} \mid \omega_{3}>0\right\}$

$$
\left.S_{+}^{2} \times \mathbb{R}^{2} \times(0, \infty) \times(\mathbb{R} \backslash 0) \ni(\omega, \mathbf{z}, r, \alpha) \mapsto\left(\mathbf{z}, r, \mathbf{z}+r \omega ; \alpha\left(\omega^{\prime} \mathbf{d z}+\mathbf{d} \mathbf{r}-\omega \mathbf{d x}\right)\right)\right)
$$

after factoring. Using these coordinates it is easy to show that the projection $\Pi_{L}: \mathcal{C} \rightarrow T^{*} Y \backslash \mathbf{0}$ is an injective immersion. This is the Bolker assumption and it implies that $R^{*} \phi R$ is a pseudodifferential operator $[10,9]$. Note that if we considered $\mathbf{x} \in \mathbb{R}^{3}$ we would need to enlarge $\mathcal{C}$ so that in coordinates (3.6), we would need to include $\omega \in S_{0}^{2}=\left\{\tau \in S^{2} \mid \tau_{3}=0\right\}$ and $\Pi_{L}$ is not an immersion above such points.

\footnotetext{
${ }^{1}$ Note that in formula (4.7) of [21], $r \mathbf{d r}$ should be $2 r \mathbf{d r}$.
} 
Using (3.5) one sees that the projection to $T^{*} \mathbb{R}_{+}^{3} \backslash \mathbf{0}, \Pi_{R}: \mathcal{C} \rightarrow T^{*} \mathbb{R}_{+}^{3} \backslash \mathbf{0}$, is also injective and for $(\mathbf{x}, \xi \mathbf{d x}) \in T^{*} \mathbb{R}_{+}^{3} \backslash \mathbf{0}, \xi_{3} \neq 0$, we have

$$
\begin{aligned}
\Pi_{R}^{-1}(\mathbf{x}, \xi \mathbf{d} \mathbf{x}) & =\left(\mathbf{z}(\mathbf{x}, \xi), r(\mathbf{x}, \xi), \mathbf{x} ; \alpha(\xi)\left(\omega^{\prime}(\xi) \mathbf{d} \mathbf{z}+\mathbf{d} \mathbf{r}-\omega(\xi) \mathbf{d} \mathbf{x}\right)\right), \\
\mathbf{z}(\mathbf{x}, \xi) & =\left(\mathbf{x}-\frac{x_{3}}{\xi_{3}} \xi\right)^{\prime}, \\
r(\mathbf{x}, \xi) & =\frac{x_{3}}{\left|\xi_{3}\right|}|\xi|, \\
\alpha(\xi) & =-\frac{\xi_{3}|\xi|}{\left|\xi_{3}\right|}, \quad \omega(\xi)=\frac{\xi_{3}}{\left|\xi_{3}\right||\xi|} \xi \in S_{+}^{2} .
\end{aligned}
$$

Since $\xi$ must be normal to the sphere $S(\mathbf{z}, r), \xi$ must be parallel $\mathbf{x}-(\mathbf{z}, 0)$. This explains (3.7). A calculation using (3.7) and the fact $x_{3}>0$ justifies (3.8). Finally, because $\xi_{3} \neq 0$ and $\omega_{3}(\xi)$ must be positive (if we require $\omega \in S_{+}^{2}$ ), (3.7) and (3.8) are used to prove (3.9).

To calculate the symbol of $\Lambda$ one follows the outline in [20]. We let $Z=\{(\mathbf{z}, r, \mathbf{x})||(\mathbf{z}, 0)-\mathbf{x}) \mid=r\}$ be the incidence relation of all spheres and points such that the point $\mathbf{x}$ is on the sphere $S(\mathbf{z}, r)$. We have already chosen measure $d m=d \mathbf{x}$ on $\mathbb{R}_{+}^{3}$ and $d n=4 \pi r^{2} d r d \mathbf{z}$ on $Y$. We choose measure on $Z$ to be $d \mu=\sqrt{\phi(\mathbf{z}, r)} r^{2} d r d \mathbf{z} d \omega$. As done by Guillemin [8] one uses these measures to define measures for the Radon transform and its dual. This gives measure on $S(\mathbf{z}, r)$ as $\frac{d \mu}{d n}=\frac{\sqrt{\phi(\mathbf{z}, r)}}{4 \pi} d \omega$ and the measure for the backprojection is $\frac{d \mu}{d \mathbf{x}}=\sqrt{\phi(\mathbf{z}, r)} \mathbf{d z}$ and so the Radon transform defined by this theory is $R^{\prime}=\sqrt{\phi} R$ and the dual transform is $\left(R^{\prime}\right)^{*}=R^{*} \sqrt{\phi}$. Therefore, $\left(R^{\prime}\right)^{*} R^{\prime}=R^{*} \phi R$, and this justifies our choices of $d x, d n$, and $d \mu$.

The next part of the calculation is to write $I_{Z}$, integration over $Z$, as a Fourier integral distribution. To do this one chooses coordinates so that $Z$ is locally defined by $w=0$ where coordinates on $Z$ are $(\tilde{z}, w)$. One then follows the mathematics on p. 337 [20] to calculate the symbol of $I_{Z}$ as a Fourier integral distribution as in the calculation of (15) in that article. One calculates that it is

$$
\sigma\left(I_{Z}\right)=\frac{(2 \pi)^{2} \phi(\mathbf{z}, r) d x d z d \eta}{(4 \pi) r^{2} \Pi_{R}^{*}\left(\left|\sigma_{\mathbb{R}^{3}}\right|^{3 / 2}\right) \Pi_{L}^{*}\left(\left|\sigma_{Y}\right|^{3 / 2}\right)}\left(\Pi_{R}^{-1}(\mathbf{x}, \xi \mathbf{d} \mathbf{x})\right)
$$

where $\sigma_{\mathbb{R}^{3}}$ and $\sigma_{Y}$ are the canonical symplectic forms on $T^{*} \mathbb{R}^{3}$ and $T^{*} Y$. To calculate the pullbacks in (3.10) one lets $\lambda=\Pi_{R}^{-1}(\mathbf{x}, \xi \mathbf{d} \mathbf{x})$ as given in (3.7)-(3.9) and one chooses a basis of $T_{\lambda} \mathcal{C}$ using the coordinates (3.6). Then, using (3.10) and this calculation of the pullbacks, we get

$$
\sigma\left(R^{*} \phi R\right)(\mathbf{x}, \xi)=\frac{2 \pi i \phi\left(\left(\mathbf{x}-\frac{x_{3}}{\xi_{3}} \xi\right)^{\prime}, \frac{x_{3}|\xi|}{\left|\xi_{3}\right|}\right)}{\left|\xi_{3}\right||\xi|}
$$

and composing with $-\partial_{x_{3}} \Delta$, which has symbol $-i \xi_{3}|\xi|^{2}$ gives us the final result (3.2). Finally, one should note that there is a Maslov symbol ([12] which is discussed in the first full paragraph in [20, p. 338]) but it must be constant because the naively calculated symbol at the start of the proof can be defined as a function (see also the discussion on [20, p. 338]). Note that different conventions for the definition of symbol can result in different constants in (3.2), but our conventions are chosen so as to agree with the naive calculations at the start of this proof.

Remark 3.3. We will now use the symbol calculation in the proof of Theorem 3.2 to explain why $\Lambda$ is smoothing off of the set $C(\mathbf{x})$ (3.3). It is clear from the definition of $C(\mathbf{x})$ that the symbol $\sigma(\Lambda)$ is zero off of $C(\mathbf{x})$, but in general this implies only that $\Lambda$ smooths one degree more off of $C(\mathbf{x})$ 
than on $C(\mathbf{x})$. Let $\mathbf{x} \in \mathbb{R}_{+}^{3}$ and $\xi \notin C(\mathbf{x})$. Then $\Pi_{L}\left(\Pi_{R}^{-1}(\mathbf{x}, \xi \mathbf{d} \mathbf{x})\right)=(\mathbf{z}, r, \eta)$ for some $\eta \in T_{(\mathbf{z}, r)}^{*} Y$. However, since $\xi \notin C(\mathbf{x})$, by (3.7)-(3.9), $\phi(\mathbf{z}, r)$ is zero. Therefore, $\phi R f(\mathbf{z}, r)=0$ is $C^{\infty}$ and so $(\mathbf{z}, r, \eta) \notin \mathrm{WF}(\phi R f)$. This shows that $(\mathbf{x}, \xi \mathbf{d} \mathbf{x})=\Pi_{R}\left(\Pi_{L}^{-1}\left(\Pi_{L}\left(\Pi_{R}^{-1}(\mathbf{x}, \xi \mathbf{d} \mathbf{x})\right)\right)\right)=\Pi_{R}\left(\Pi_{L}^{-1}(\mathbf{z}, r, \eta)\right)$ is not in $\mathrm{WF}\left(R^{*}(\phi R f)\right)$. Here we are using the fact $\Pi_{L}$ and $\Pi_{R}$ are injective as well as composition calculus for Fourier integral operators [12]: for $A \subset T^{*} \mathbb{R}_{+}^{3} \backslash \mathbf{0}, \mathcal{C} \circ A=\Pi_{L}\left(\Pi_{R}^{-1}(A)\right)$ and for $B \subset T^{*} Y, \mathcal{C}^{t} \circ B=\Pi_{R}\left(\Pi_{L}^{-1}(B)\right)$. Here we are using the following notation [12]: $\mathcal{C}^{t}$ is $\mathcal{C}$ but with the $T^{*} \mathbb{R}^{3}$ and $T^{*} Y$ coordinates reversed, and for $A \subset T^{*} \mathbb{R}^{3}$,

$$
\mathcal{C} \circ A=\left\{(\mathbf{z}, r, \eta) \in T^{*} Y \mid \exists(\mathbf{x}, \xi) \in A, \text { with }(\mathbf{z}, r, \mathbf{x} ; \eta, \xi) \in \mathcal{C}\right\} .
$$

Therefore, $\Lambda$ is smoothing in direction $(\mathbf{x}, \xi \mathbf{d} \mathbf{x})$.

\section{The Approximate Inverse: Mollifier $e_{\mathbf{p}, s, k}$ And Reconstruction Kernel $\psi_{\mathbf{p}, s, k}$}

For an implementation of our local reconstruction operator $\Lambda$ we need to stabilize its numerical evaluation. Several approaches are possible. We follow ideas of the approximate inverse [15] as it provides a general and well-developed framework for the stable solution of operator equations of the first kind, see e.g. [22, 23, 24].

Instead of computing $\Lambda f(\mathbf{p}), \mathbf{p} \in \mathbb{R}_{+}^{3}$, directly we want to recover the smoothed version

$$
\left\langle\Lambda f, e_{\mathbf{p}, s, k}\right\rangle_{L^{2}\left(\mathbb{R}^{3}\right)}
$$

where

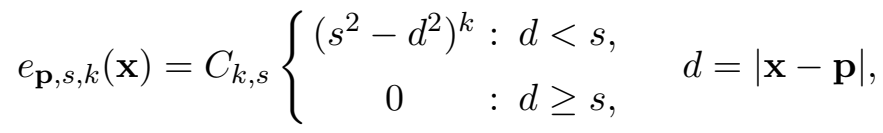

serves as mollifier with $s, k>0$ and

$$
C_{k, s}=\left(\int_{B_{s}(\mathbf{p})}\left(s^{2}-d^{2}\right)^{k} d V\right)^{-1}=\frac{\Gamma(k+5 / 2)}{\pi^{3 / 2} \Gamma(k+1) s^{3+2 k}} .
$$

Observe that

$$
\int_{\mathbb{R}^{3}} e_{\mathbf{p}, s, k}(\mathbf{x}) \mathrm{d} \mathbf{x}=1 \quad \text { and } \quad \operatorname{supp} e_{\mathbf{p}, s, k}=B_{s}(\mathbf{p}) .
$$

Further, the inner product (4.1) can be expressed as a convolution integral:

$$
\Lambda f * e_{\mathbf{0}, s, k}(\mathbf{p})=\left\langle\Lambda f, e_{\mathbf{p}, s, k}\right\rangle_{L^{2}\left(\mathbb{R}^{3}\right)} .
$$

The parameter $s>0$ scales the mollifier and plays the role of a regularization parameter: the larger $s$ the smoother the reconstruction. In the sequel we implicitly assume $s<p_{3}$ yielding $\operatorname{supp} e_{\mathbf{p}, s, k} \subset \mathbb{R}_{+}^{3}$ for $\mathbf{p} \in \mathbb{R}_{+}^{3}$. Note that $k$ is merely a design parameter.

In the following theorem we give analytically a so-called reconstruction kernel allowing the computation of $\left\langle\Lambda f, e_{\mathbf{p}, s, k}\right\rangle_{L^{2}\left(\mathbb{R}^{3}\right)}$ from the spherical means of $f$.

Theorem 4.1. We have that

$$
\left\langle\Lambda f, e_{\mathbf{p}, s, k}\right\rangle_{L^{2}\left(\mathbb{R}^{3}\right)}=\left\langle R f, \psi_{\mathbf{p}, s, k}\right\rangle_{L^{2}\left(\mathbb{R}^{2} \times\left[0, \infty\left[, r^{2} \mathrm{~d} \mathbf{z d} r\right)\right.\right.}
$$


with reconstruction kernel

$$
\begin{aligned}
\psi_{\mathbf{p}, s, k}(\mathbf{z}, r)=\phi(\mathbf{z}, r) \frac{C_{k, s} k p_{3} A^{k-2}}{L} & {\left[(2 k+1) A\left[\frac{1}{L k}\left(k-2+\frac{B}{2 r L}\right)-\frac{1}{r}\right]\right.} \\
& \left.+2(k-1) s^{2}\left[\frac{1}{r}-\frac{1}{L(k-1)}\left(k-3+\frac{B}{2 r L}\right)\right]\right]
\end{aligned}
$$

for $r \in[L-s, L+s]$ where

$$
L=|(\mathbf{z}, 0)-\mathbf{p}|, \quad A=s^{2}-(L-r)^{2} \text { and } B=(r+L)^{2}-s^{2} .
$$

For $r \notin[L-s, L+s]: \psi_{\mathbf{p}, s, k}(\mathbf{z}, r)=0$.

The proof of the theorem can be found in Appendix A.

\section{Reconstructions}

In this section we give numerical reconstructions using the approximate inverse. We will also interpret the results in terms of the microlocal properties of $R$ and $\Lambda$ that were given in Section 3 .

We want to approximate

$$
\Lambda f(\mathbf{p}) \approx\left\langle\Lambda f, e_{\mathbf{p}, s, k}\right\rangle_{L^{2}\left(\mathbb{R}^{3}\right)}=\left\langle R f, \psi_{\mathbf{p}, s, k}\right\rangle_{L^{2}\left(\mathbb{R}^{2} \times\left[0, \infty\left[, r^{2} d \mathbf{z} d r\right)\right.\right.}
$$

from the discrete data

$$
g(i, j, k)=R f\left(\mathbf{z}_{i, j}, r_{k}\right), \quad i, j=1, \ldots, N_{\mathrm{z}}, k=1, \ldots, N_{\mathrm{r}},
$$

where

$$
\left\{\mathbf{z}_{i, j}\right\} \subset\left[-z_{\max }, z_{\max }\right]^{2} \text { and } \quad\left\{r_{k}\right\} \subset\left(0, r_{\max }\right]
$$

are Cartesian grids with uniform step sizes $h_{\mathrm{z}}$ and $h_{\mathrm{r}}$, respectively. A straightforward discretization of the triple integral on the right of (5.1) yields

$$
\begin{aligned}
\Lambda f(\mathbf{p}) \approx \widetilde{\Lambda} f(\mathbf{p}) & :=h_{\mathrm{z}}^{2} h_{\mathrm{r}} \sum_{i=1}^{N_{\mathrm{z}}} \sum_{j=1}^{N_{\mathrm{z}}} \sum_{k=1}^{N_{\mathrm{r}}} g(i, j, k) \psi_{\mathbf{p}, s, k}\left(\mathbf{z}_{i, j}, r_{k}\right) r_{k}^{2} \\
& =h_{\mathrm{z}}^{2} h_{\mathrm{r}} \sum_{i=1}^{N_{\mathrm{z}}} \sum_{j=1}^{N_{\mathrm{z}}} \sum_{r_{k} \in \mathcal{L}_{i, j}(\mathbf{p})} g(i, j, k) \psi_{\mathbf{p}, s, k}\left(\mathbf{z}_{i, j}, r_{k}\right) r_{k}^{2}
\end{aligned}
$$

with $\mathcal{L}_{i, j}(\mathbf{p})=[L-s, L+s]$ and $L=\left|\left(\mathbf{z}_{i, j}, 0\right)-\mathbf{p}\right|$.

For our numerical computations presented in this section we have chosen the following cutoff function $\phi(2.2)$. Given $0<\delta<\bar{M}$ and $\bar{T}>0$ we define

$$
\phi(\mathbf{z}, r)=\alpha(\mathbf{z}) \beta(r)
$$

where

$$
\beta(r)=\left\{\begin{array}{cc}
0 & : r \leq \delta \text { or } r \geq \bar{M}+1 \\
1 & : \quad 2 \delta \leq r \leq \bar{M} \\
p(r, \bar{M}): & \bar{M}<r<\bar{M}+1 \\
q(r, \delta): & \delta<r<2 \delta
\end{array}\right.
$$




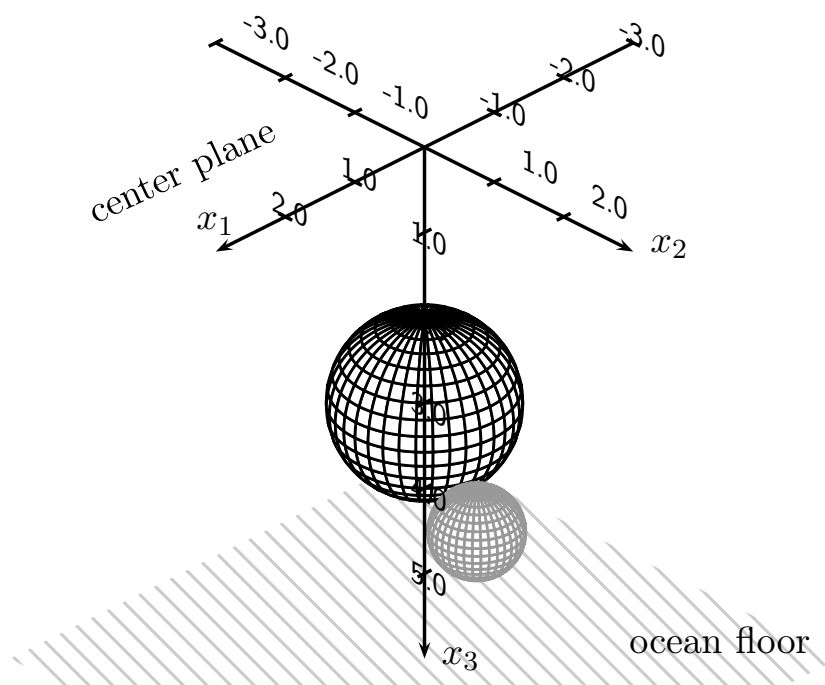

Figure 1. Visualization of the function (5.4) to be reconstructed. The two balls slightly intersect.

with

$$
p(r, \bar{M})=\frac{u(\bar{M}+1-r)}{u(\bar{M}+1-r)+u(r-\bar{M}-1 / 2)}, \quad q(r, \delta)=\frac{u(r / \delta-1)}{u(r / \delta-1)+u(2-r / \delta)}
$$

and

$$
u(x)=\left\{\begin{array}{cl}
\exp (-1 / x) & : x>0 \\
0 & : x \leq 0
\end{array}\right.
$$

Further,

$$
\alpha(\mathbf{z})=\tilde{\alpha}\left(z_{1}\right) \tilde{\alpha}\left(z_{2}\right) \text { and } \tilde{\alpha}(x)=\left\{\begin{array}{ccc}
1 & : & |x|<\bar{T} \\
p(|x|, \bar{T}) & : \bar{T} \leq|x| \leq \bar{T}+1 \\
0 \quad & : & |x|>\bar{T}+1
\end{array}\right.
$$

Thus,

$$
\phi \in C^{\infty}\left(\mathbb{R}^{3}\right), \operatorname{supp} \phi \subset[-\bar{T}-1, \bar{T}+1]^{2} \times[\delta, \bar{M}+1], \text { and }\left.\phi\right|_{[-\bar{T}, \bar{T}]^{2} \times[2 \delta, \bar{M}]}=1 .
$$

We always set $\bar{M}:=r_{\max }-1, \delta:=0.01$, and $\bar{T}=z_{\max }-1$.

The function $f: \mathbb{R}_{+}^{3} \rightarrow \mathbb{R}$ to be reconstructed is a superposition of three indicator functions given by

$$
f=\chi_{B_{1}(0,0,3)}-\chi_{B_{0.5}(0.25,1,4)}+0.3 \chi_{x_{3} \geq 6}
$$

whose Sonar transform can be calculated analytically. The rightmost indicator function models a flat ocean floor at $x_{3}=6$, see Figure 1 for a visualization.

In our first set of experiments we will demonstrate which singularities of $f$ can be detected depending on the available data. To this end we note that the wavefront set of $f$ (compare (3.1)) 
is

$$
\begin{aligned}
& \mathrm{WF}(f)=\left\{(\mathbf{x}, \xi) \mid \mathbf{x} \in \partial B_{1}(0,0,3), \xi=\lambda(\mathbf{x}-(0,0,3)), \lambda \neq 0\right\} \\
& \begin{aligned}
\cup\left\{(\mathbf{x}, \xi) \mid \mathbf{x} \in \partial B_{0.25}(0.25,1,4), \quad \xi=\lambda(\mathbf{x}-(0.25,1,4)), \quad \lambda \neq 0\right\} \\
\cup\left\{(\mathbf{x}, \xi) \mid x_{3}=6, \quad \xi_{1}=\xi_{2}=0, \quad \xi_{3} \neq 0\right\},
\end{aligned}
\end{aligned}
$$

that is, the wavefront set consists of all pairs $(\mathbf{x}, \xi)$ where $\mathbf{x}$ is on the boundary of either one of the two balls or of the ocean floor and $\xi$ is normal to the boundary at this point. ${ }^{2}$

Figure 2 displays cross sections $\widetilde{\Lambda} f(0.25, \cdot, \cdot)$ for three different sets of limited data: $z_{\max }=3$ (top left), $z_{\max }=6$ (top right), and $z_{\max }=12$ (bottom). Further, $r_{\max }=10$ in all three settings. All cross sections have been computed from $N_{\mathrm{z}}^{2} N_{\mathrm{r}}=301^{2} \cdot 250=22650250$ spherical means.

To understand the extent to which our results from section 3 are reflected in our reconstructions, we inspect the set $C(\mathbf{x})(3.3)$ on which $\Lambda$ is elliptic of order one. The wavefront $(\mathbf{x}, \xi) \in \mathrm{WF}(f)$ will be visible in $\Lambda f$ (or $\widetilde{\Lambda} f$ ) if $\xi \in C(\mathbf{x})$, that is, $\xi_{3} \neq 0$ and

$$
\frac{x_{i}-z_{\max }}{x_{3}}<\frac{\xi_{i}}{\xi_{3}}<\frac{x_{i}+z_{\max }}{x_{3}}, i=1,2, \quad \text { and } \quad \frac{\delta}{x_{3}}<\frac{|\xi|}{\left|\xi_{3}\right|}<\frac{r_{\max }}{x_{3}} .
$$

Thus, wavefronts for which $\xi$ has dominant horizontal components $\left(\left|\xi_{3}\right|\right.$ is small compared to $\left.|\xi|\right)$ will not be recovered. The visible wavefronts have dominant vertical components and the smaller $z_{\max }$ and $r_{\max }$ are and the larger $x_{3}$ is, the more dominant the vertical components have to be to be visible.

This fact is illustrated by the reconstructions shown in Figures 2. With increasing $z_{\max }$ (top left, top right, bottom) more and more singularities of $f$ are recovered. In the bottom reconstruction only the singularities with almost horizontal directions are missing. The ocean floor $\left\{(\mathbf{x}, \xi) \mid x_{3}=\right.$ $\left.6, \xi_{1}=\xi_{2}=0, \xi_{3} \neq 0\right\}$ is visible because we have

$$
\frac{x_{i}-z_{\max }}{6}<0<\frac{x_{i}+z_{\max }}{6}\left(\Leftrightarrow-z_{\max }<x_{i}<z_{\max }\right) \quad \text { and } \quad \frac{0.01}{6}<1<\frac{10}{6} .
$$

In Figure 3 we again display $\widetilde{\Lambda} f(0.25, \cdot, \cdot)$ with $z_{\max }=3$, however, with clearly reduced maximal radii: $r_{\max }=4.5$ and $r_{\max }=5.5$. The ocean floor is not recovered by either reconstruction. For $r_{\max }=4.5$ even the bottom hemisphere of the smaller ball is missing and a strong artefact corrupts the reconstruction.

\footnotetext{
${ }^{2}$ The two boundary spheres intersect in a small circle. At each intersection point are two "singularity directions" corresponding to normals to the two spheres.
} 

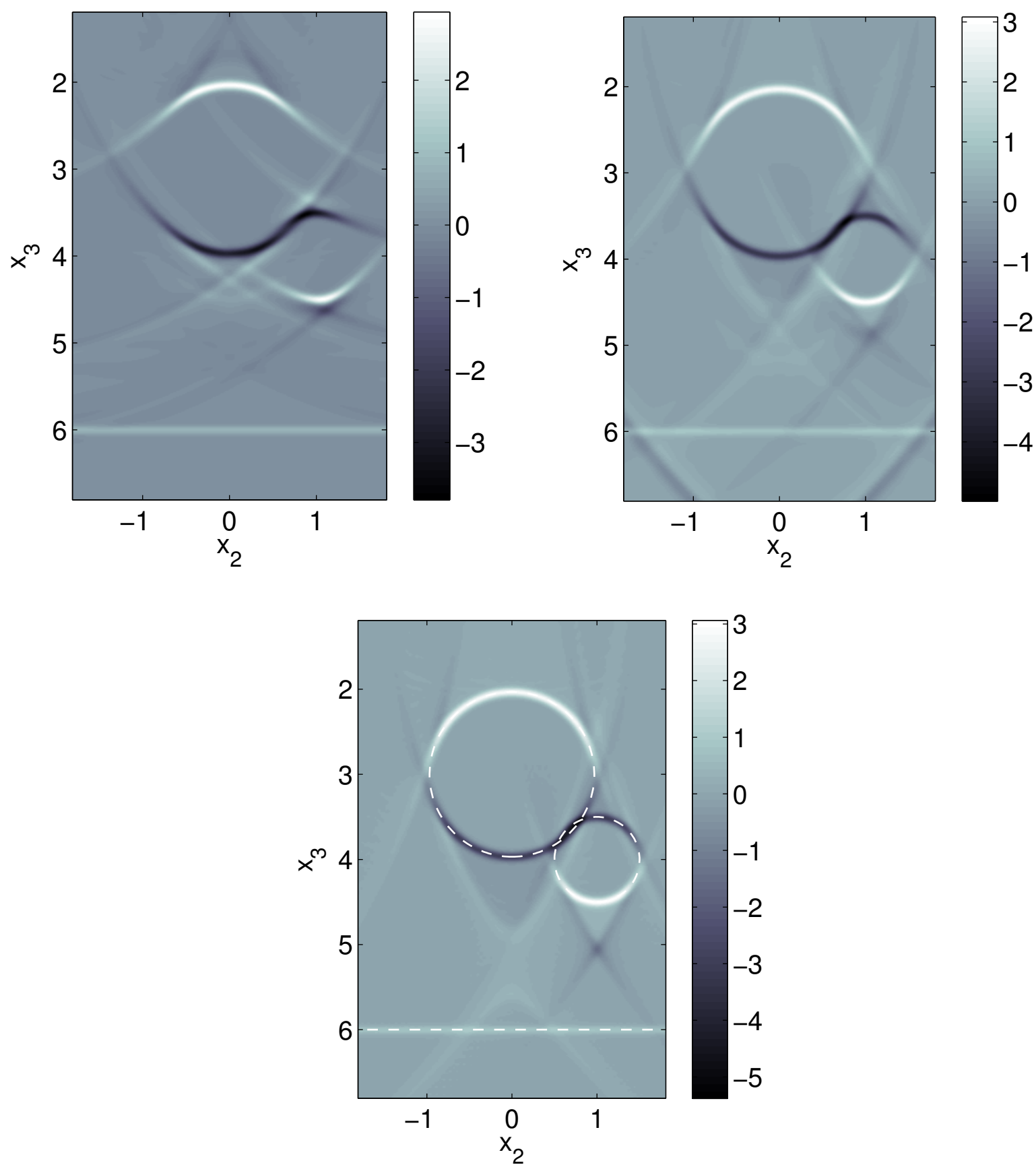

Figure 2. Reconstructions $\widetilde{\Lambda} f(0.25, \cdot, \cdot), f$ from (5.4), where $r_{\max }=10$ and $z_{\max }=$ 3 (top left), $z_{\max }=6$ (top right), and $z_{\max }=12$ (bottom) with $N_{\mathrm{z}}=301$ and $N_{\mathrm{r}}=250$, see (5.2) and (5.3). The parameters used for the reconstruction kernel are $s=0.8$ and $k=3$. The dashed white lines in the bottom reconstruction indicate the singular support of $f$ and are not part of the reconstruction. 

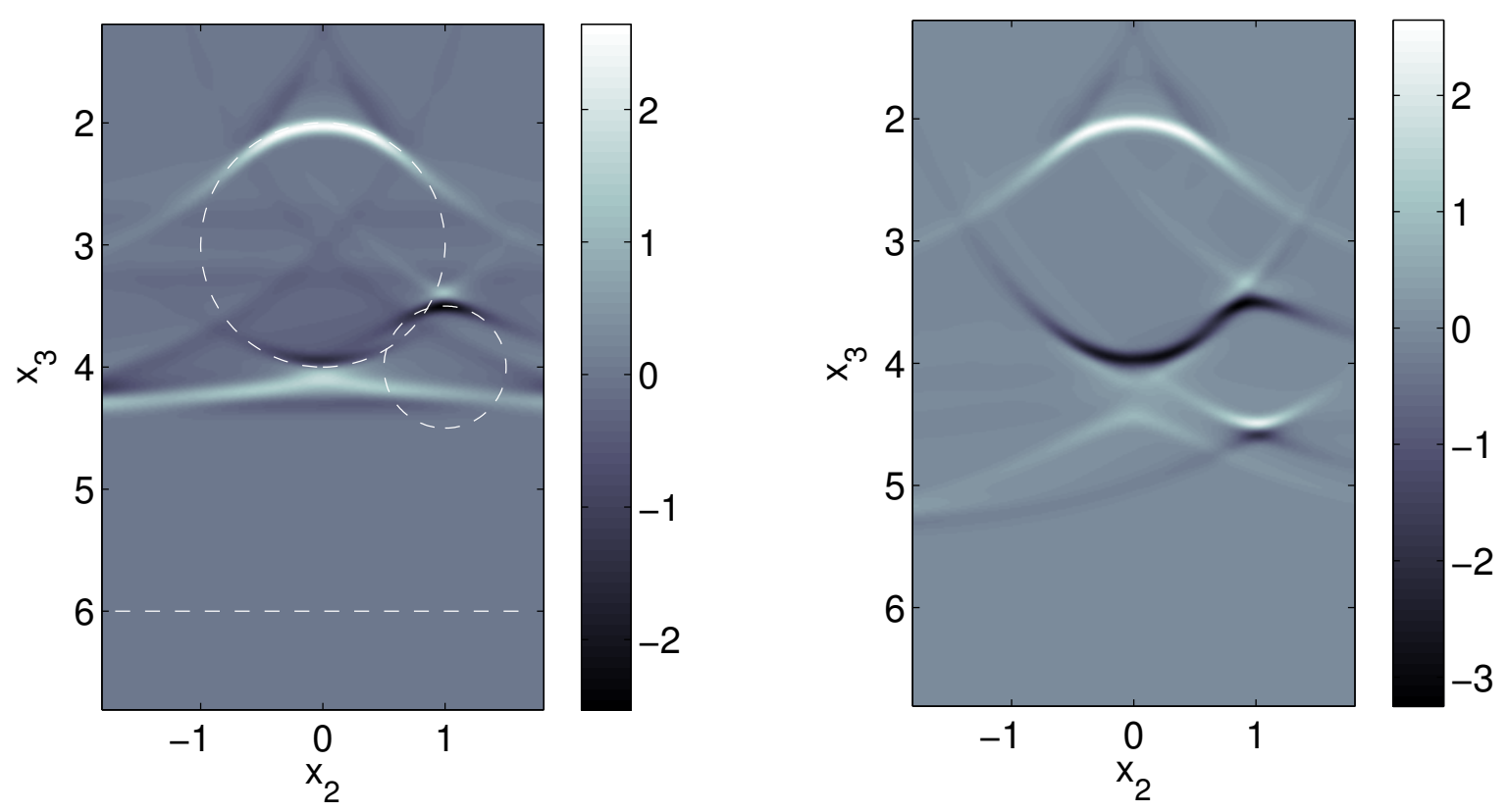

Figure 3. Cross sections $\widetilde{\Lambda} f(0.25, \cdot, \cdot), f$ from (5.4), where $r_{\max }=4.5$ (left) and $r_{\max }=5.5$ (right). Further, $z_{\max }=3, N_{\mathrm{z}}=301$ and $N_{\mathrm{r}}=200$, see (5.2) and (5.3). The parameters used for the reconstruction kernel are $s=0.8$ and $k=3$. The dashed white lines in the left reconstruction indicate the singular support of $f$ and are not part of the reconstruction. 
To prove that we really perform fully 3D reconstructions we display several cross sections $\widetilde{\Lambda} f\left(x_{1, i}, \cdot, \cdot\right)$ for $x_{1, i}=-1+0.25 i, i=0, \ldots, 8$, in Figure 4 . Here we like to emphasize the following observation: the boundaries of the two balls in the different cross sections are reconstructed with different intensity (note the different gray scales). The reason for this fact is that the 3Ddirection of the corresponding wavefront does not agree with the 2D-normal on the ball in the displayed cross section. ${ }^{3}$ The more the wavefront direction differs from the $2 \mathrm{D}$-normal in the cross section the less pronounced is the singularity in the respective cross section.

We finish the numerics section by demonstrating how the algorithm performs with noisy data. To this end we perturb the exact data $g(5.2)$ according to

$$
g^{\varepsilon}(i, j, k)=g(i, j, k)+\varepsilon\|g\|_{\star} \frac{\operatorname{noise}(i, j, k)}{\|\operatorname{noise}\|_{\star}}, \quad \varepsilon>0,
$$

where noise is an $N_{\mathrm{z}} \times N_{\mathrm{z}} \times N_{\mathrm{r}}$ array of uniformly distributed random numbers ${ }^{4}$ with values in $[-1,1]$ and where the discrete norm

$$
\|g\|_{\star}^{2}:=h_{\mathrm{z}}^{2} h_{\mathrm{r}} \sum_{i=1}^{N_{\mathrm{z}}} \sum_{j=1}^{N_{\mathrm{z}}} \sum_{k=1}^{N_{\mathrm{r}}}|g(i, j, k)|^{2} r_{k}^{2}
$$

approximates the norm in $L^{2}\left(\left[-\mathbf{z}_{\max }, \mathbf{z}_{\max }\right]^{2} \times\left[0, r_{\max }\right], r^{2} \mathrm{~d} \mathbf{z} \mathrm{d} r\right)$. We have that

$$
\frac{\left\|g-g^{\varepsilon}\right\|_{\star}}{\|g\|_{\star}} \leq \varepsilon
$$

Thus, $\varepsilon$ measures the relative noise. In all experiments below we worked with $\varepsilon=3 \%$.

First, the smoothing or regularizing effect of the scaling parameter $s$ is illustrated. Figure 5 contains reconstructions of a cross section $\left(x_{1}=0.25\right)$ from the same perturbed data for 4 different scaling parameters. As $s$ increases the noise gets reduced at the price of blurred and fuzzy contours.

Finally, in Figure 6 we present the same cross sections of $\widetilde{\Lambda} f$ as in Figure 4, however, reconstructed from noisy data with scaling parameter $s=1.6$.

\footnotetext{
${ }^{3}$ We have two exceptions: for $x_{1}=0$ the 2 D-normals on the large ball in the cross section agree with the corresponding directions of the wavefronts. The same holds true for the small ball at $x_{1}=0.25$.

${ }^{4}$ In all computations we used the same noise array.
} 

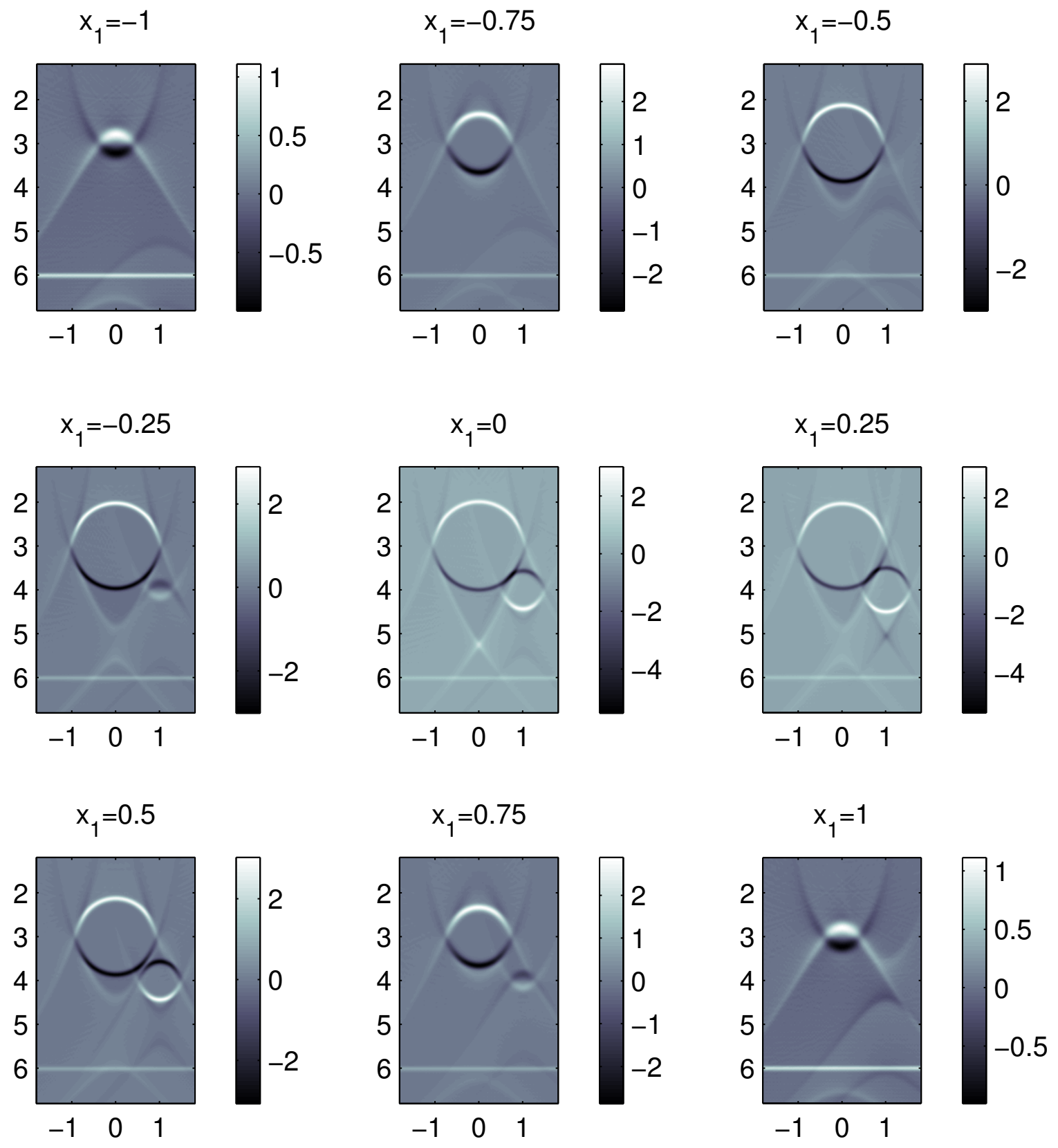

Figure 4. Cross sections $\widetilde{\Lambda} f\left(x_{1}, \cdot, \cdot\right), f$ from (5.4), for several $x_{1}$ 's. Here, $r_{\max }=10$, $z_{\max }=12, N_{\mathrm{z}}=301$ and $N_{\mathrm{r}}=250$, see (5.2) and (5.3). The parameters used for the reconstruction kernel are $s=0.8$ and $k=3$. Please note the different gray scales for each reconstruction. 

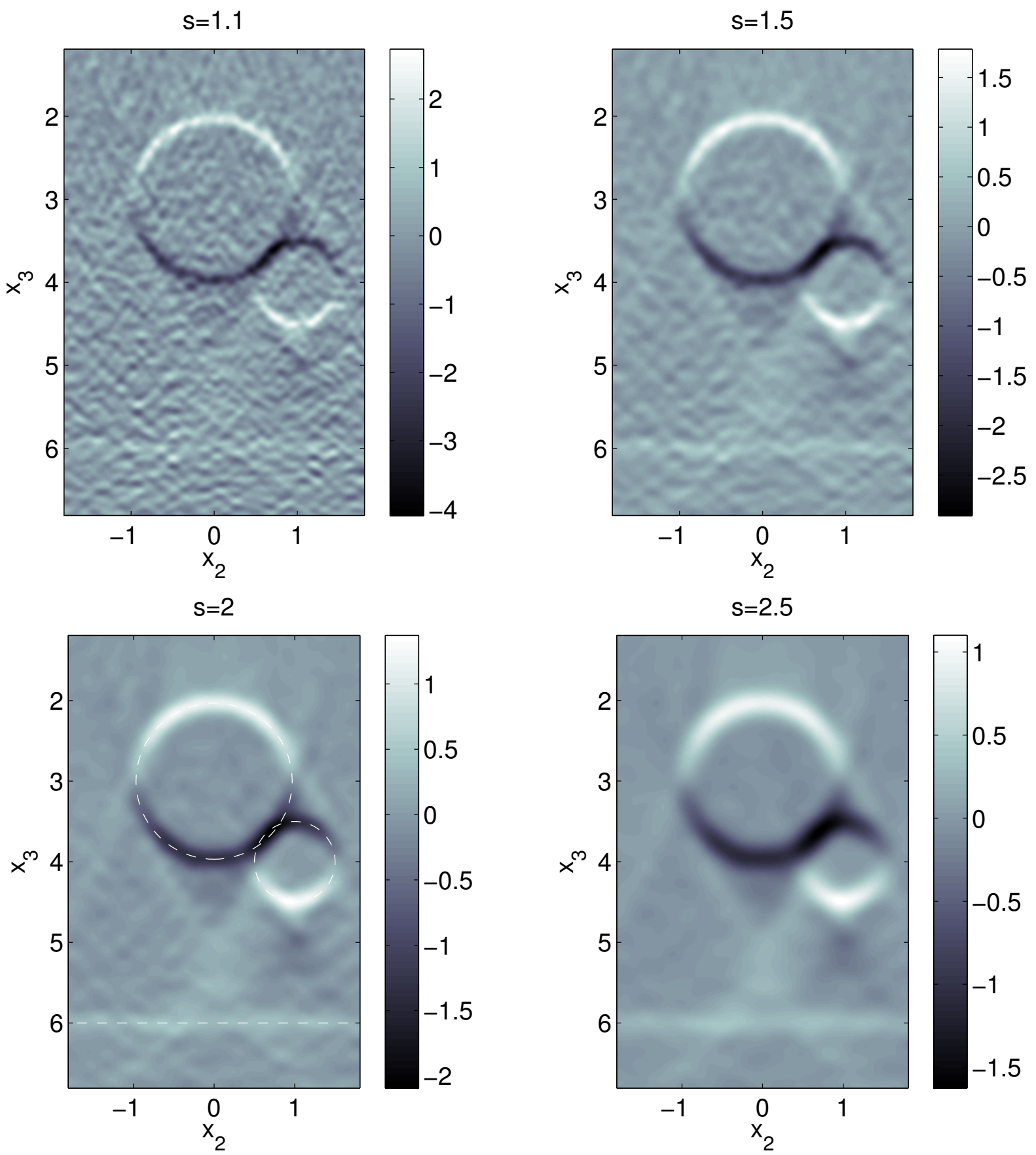

FigURE 5. Reconstructions $\widetilde{\Lambda} f(0.25, \cdot, \cdot), f$ from $(5.4)$, under $3 \%$ relative noise for different scaling parameter: $s=1.1$ (top left), $s=1.5$ (top right), $s=2$ (bottom left), and $s=2.5$ (bottom right). Further, $r_{\max }=10, z_{\max }=12, N_{\mathrm{z}}=301$, $N_{\mathrm{r}}=250$, see (5.2) and (5.3), and $k=3$. The dashed white lines in the bottom left reconstruction indicate the singular support of $f$ and are not part of the reconstruction. 

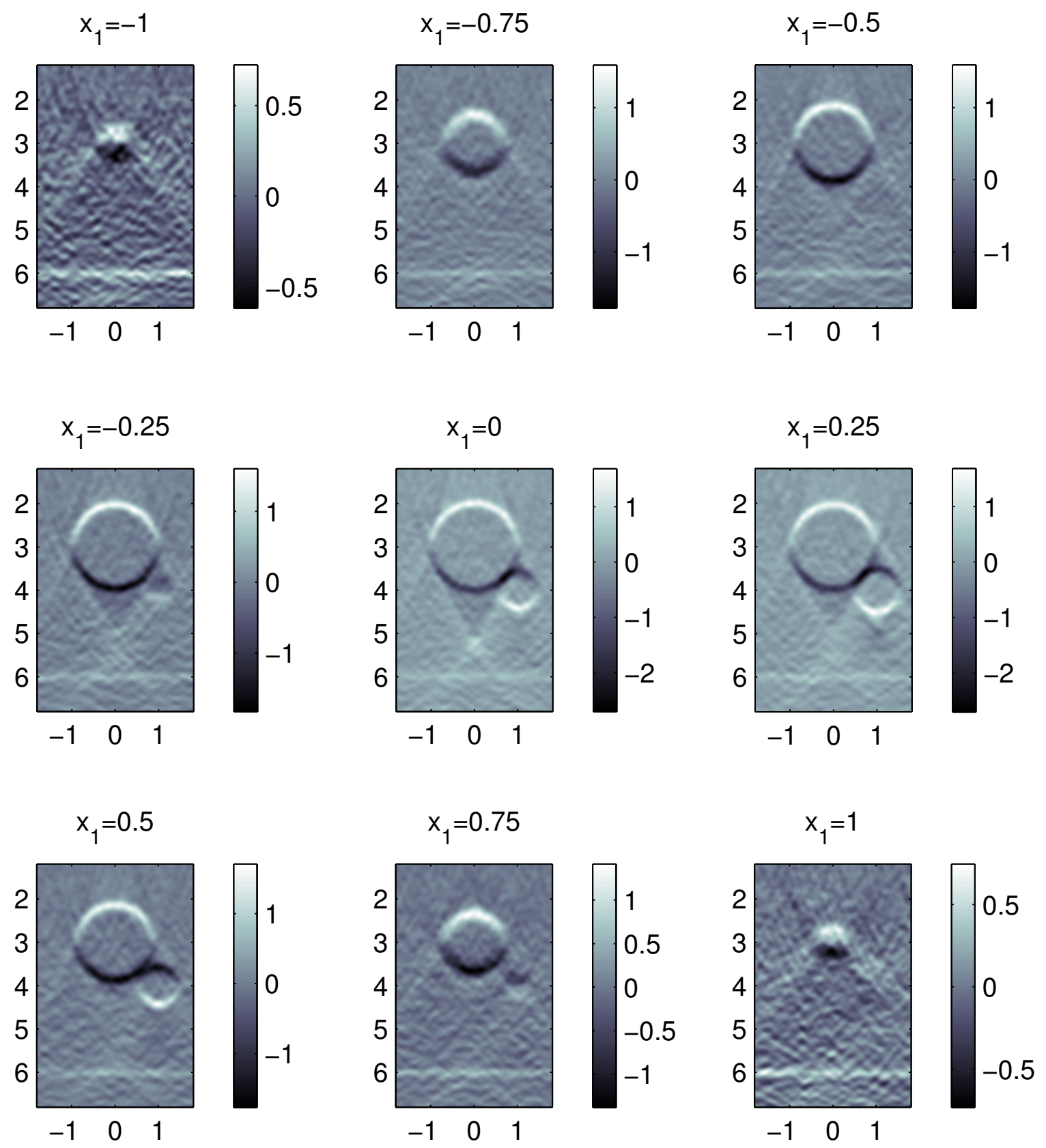

FiguRE 6. Cross sections $\widetilde{\Lambda} f\left(x_{1}, \cdot, \cdot\right), f$ from (5.4), for several $x_{1}$ 's under $3 \%$ relative noise. Here, $r_{\max }=10, z_{\max }=12, N_{\mathrm{z}}=301$ and $N_{\mathrm{r}}=250$, see (5.2) and (5.3). The parameters used of the reconstruction kernel are $s=1.6$ and $k=3$. Please note the different gray scales for each reconstruction. 


\section{Appendix A. The proof of Theorem 4.1}

To find $\psi_{\mathbf{p}, s, k}$, we start from (4.2) and by duality, we must have

$$
\psi_{\mathbf{p}, s, k}(\mathbf{z}, r)=\phi(\mathbf{z}, r) R\left(\partial_{x_{3}} \Delta e_{\mathbf{p}, s, k}\right)(\mathbf{z}, r) .
$$

So as not to deal with too many constants, we consider an unnormalized version of $e_{\mathbf{p}, s, k}$ and define

$$
\tilde{e}_{\mathbf{p}, s, k}=e_{\mathbf{p}, s, k} / C_{k, s} \text {. }
$$

Now we state the pieces we need to prove the expression for $\psi_{\mathbf{p}, s, k}$ in Theorem 4.1, and we use (A.1).

Lemma A.1. Let $k \in \mathbb{N}, k \geq 3$. Then,

$$
\begin{aligned}
R\left(\partial_{x_{3}} \Delta \tilde{e}_{\mathbf{p}, s, k}\right)(\mathbf{z}, r) & =\left[4 k(2 k+1)(k-1)\left[R\left(x_{3} \tilde{e}_{\mathbf{p}, s, k-2}\right)-p_{3} R\left(\tilde{e}_{\mathbf{p}, s, k-2}\right)\right]\right. \\
& \left.+8 k(k-1)(k-2) s^{2}\left[p_{3} R\left(\tilde{e}_{\mathbf{p}, s, k-3}\right)-R\left(x_{3} \tilde{e}_{\mathbf{p}, s, k-3}\right)\right]\right] .
\end{aligned}
$$

For $\ell$ a nonnegative integer

$$
R \tilde{e}_{\mathbf{p}, s, \ell}(\mathbf{z}, r)=\frac{A^{\ell+1}}{4(\ell+1) r L} \quad r \in(L-s, L+s)
$$

and

$$
R\left(x_{3} \tilde{e}_{\mathbf{p}, s, \ell}\right)(\mathbf{z}, r)=\frac{p_{3} A^{\ell+1}}{4 L^{2}(\ell+1)(\ell+2)}\left(\ell+\frac{B}{2 r L}\right) \quad r \in(L-s, L+s) .
$$

where $L=|(\mathbf{z}, 0)-\mathbf{p}|, A=s^{2}-(L-r)^{2}$, and $B=(r+L)^{2}-s^{2}$ and where $r \in(L-s, L+s)$ and the functions in (A.3) and (A.4) are zero outside this interval.

The proof of (A.2) follows by a calculation and for $k=3$ noting that the derivatives are distributional derivatives (since $\tilde{e}_{\mathbf{p}, s, 0}$ is not continuous). Using (A.3) and (A.4) for various values of $\ell$ in (A.2), one proves (4.3) in Theorem 4.1.

To prove the lemma, first recall that $\mathbf{p}$ is the center of the mollifier $\tilde{e}_{\mathbf{p}, s, \ell}$. We assume the sphere we are integrating over has radius $r$ and is centered at $\mathbf{z}=\left(z_{1}, z_{2}\right)$ on the $x_{1} x_{2}$-plane. Furthermore, we denote the distance from $\mathbf{z}$ to $\mathbf{p}$ by

$$
L:=|(\mathbf{z}, 0)-\mathbf{p}| .
$$

The condition for the integral to be nonzero is $r \in(L-s, L+s)$, so assume $r$ is in this interval. Also, $L \geq p_{3}$ since $\mathbf{z}$ is on the $x_{1} x_{2}$-plane and $p_{3}>0$.

The calculation of $R\left(\partial_{x_{3}} \Delta \tilde{e}_{\mathbf{p}, s, k}\right)$ reduces to expressions involving $R\left(\tilde{e}_{\mathbf{p}, s, \ell}\right)$ and $R\left(x_{3} \tilde{e}_{\mathbf{p}, s, \ell}\right)$ for $\ell=k-3, k-2, k-1$ if one uses that $\tilde{e}_{\mathbf{p}, s, k}$ is radial about $\mathbf{p}$, and one uses the radial form of the Laplacian, $\Delta=\frac{\partial^{2}}{\partial d^{2}}+\frac{2}{d} \frac{\partial}{\partial d}$ where $d=|\mathbf{x}-\mathbf{p}|$. One uses this observation, to calculate (A.2).

We now prove (A.4) by rotating and translating the picture and then using spherical coordinates. The proof of (A.3) uses similar arguments but is simpler. If we take a point $\mathbf{v}$ on $S(\mathbf{z}, r)$ then the $x_{3}$ coordinate of $\mathbf{v}$ is simply $\mathbf{v} \cdot e_{3}$. This is the same as

$$
x_{3}=\mathbf{v} \cdot e_{3}=(\mathbf{v}-\mathbf{z}) \cdot e_{3}
$$

since $\mathbf{z}$ is on the $x_{1} x_{2}$-plane.

Let $\alpha \in(0, \pi / 2]$ be the angle between the vectors $\left(\left(\mathbf{p}^{\prime}, 0\right)-\mathbf{z}\right)$ and $(\mathbf{p}-\mathbf{z})$. Note that

$$
\sin \alpha=p_{3} / L \text {. }
$$


We make a rigid motion of $\mathbb{R}^{3}$ so that $(\mathbf{z}, 0)$ is mapped to the origin, $\mathbf{p}$ to $(0,0, L)$ and $\left(\mathbf{p}^{\prime}, 0\right)$ to the point in the $x_{1} x_{3}$-plane

$$
(0,0, L)+p_{3}(\cos \alpha, 0,-\sin \alpha) .
$$

Under this transformation, $e_{3}$ is mapped to the unit vector in the direction from $(0,0, L)-$ $x_{3}(\cos \alpha, 0,-\sin \alpha)$ to $(0,0, L)$. That is,

$$
e_{3} \mapsto(-\cos \alpha, 0, \sin \alpha) .
$$

If $\mathbf{v} \in S(\mathbf{z}, r)$ let $\tilde{\mathbf{v}}$ be the point on $S(0, r)$ to which it is mapped under this rotation. Then the $x_{3}$-coordinate of $\mathbf{v}$ is

$$
x_{3}=\mathbf{v} \cdot e_{3}=(\mathbf{v}-(\mathbf{z}, 0)) \cdot e_{3}=(\tilde{\mathbf{v}}-(0,0,0)) \cdot(-\cos \alpha, 0, \sin \alpha)
$$

since the dot product does not change under rigid motion and because under this rigid motion $e_{3}$ gets mapped to the vector in (A.6).

We can use spherical coordinates about the $x_{3}$-axis to integrate so an arbitrary point on the sphere of radius $r$ centered at the origin is

$$
(\theta, \phi) \mapsto \tilde{\mathbf{v}}=r(\cos \theta \sin \phi, \sin \theta \sin \phi, \cos \phi) .
$$

Using (A.7), we see that, in these new coordinates, $x_{3}$ is

$$
x_{3}=r(\cos \theta \sin \phi, \sin \theta \sin \phi, \cos \phi) \cdot(-\cos \alpha, 0, \sin \alpha)=-r \cos \alpha \sin \phi \cos \theta+r \cos \phi \sin \alpha .
$$

When we put this into the integral of the spherical mean, we get

$$
R\left(x_{3} \tilde{e}_{\mathbf{p}, s, \ell}\right)(\mathbf{z}, r)=\frac{1}{4 \pi} \int_{\phi=0}^{\Phi} \int_{\theta=0}^{2 \pi}\left(s^{2}-d^{2}\right)^{\ell}[-r \cos \alpha \sin \phi \cos \theta+r \cos \phi \sin \alpha] \sin \phi d \theta d \phi,
$$

where $\Phi$ is the upper limit of integration. Since $\Phi$ is the angle at the origin of the triangle with vertices the origin and $(0,0, L)$, and with sides $r, L s$, the law of cosines shows that

$$
\begin{gathered}
s^{2}=r^{2}+L^{2}-2 r L \cos \Phi, \\
\cos \Phi=\frac{L^{2}+r^{2}-s^{2}}{2 r L} .
\end{gathered}
$$

Now, we do some simple calculations. First, recall that in the integral (A.8), $\phi$ is the angle of inclination from the $x_{3}$-axis to the point being integrated, so $d^{2}=r^{2}+L^{2}-2 r L \cos \phi$ and using (A.9) we see

$$
s^{2}-d^{2}=2 r L(\cos \phi-\cos \Phi)
$$

and integral (A.8) becomes

$$
R\left(x_{3} \tilde{e}_{\mathbf{p}, s, \ell}\right)(\mathbf{z}, r)=\frac{(2 r L)^{\ell}}{4 \pi} \int_{\phi=0}^{\Phi} \int_{\theta=0}^{2 \pi}[-r \cos \alpha \sin \phi \cos \theta+r \cos \phi \sin \alpha](\cos \phi-\cos \Phi)^{\ell} \sin \phi d \theta d \phi .
$$

First, note that after we integrate in $\theta$, the first term in brackets drops out, so we are left with

$$
R\left(x_{3} \tilde{e}_{\mathbf{p}, s, \ell}\right)(\mathbf{z}, r)=\frac{r(2 r L)^{\ell}}{2} \int_{\phi=0}^{\Phi}[\cos \phi \sin \alpha](\cos \phi-\cos \Phi)^{\ell} \sin \phi d \phi .
$$

Now, if we make the substitution $u=\cos \phi-\cos \Phi$ (and $\cos \phi=u+\cos \Phi)$ and use the expression (A.5) for $\sin \alpha$, then we get (A.4).

If one goes through a calculation using the same steps but without the factor of $x_{3}$, then one gets (A.3). In both (A.3) and (A.4), it helps to simplify $1-\cos \Phi$ and $1+\cos \Phi$ using (A.9). 


\section{REFERENCES}

[1] M. Agranovsky and P. Kuchment and L. Kunyansky, On reconstruction formulas and algorithms for the thermoacoustic tomography, in Photoacoustic imaging and spectroscopy, pp. 89-101, editor L.H. Wang, CRC Press, 2009.

[2] L-E. Andersson, On the determination of a function from spherical averages, SIAM J. Math. Anal. 19 (1988), $214-232$.

[3] J. Cohen and H. Bleistein, Velocity inversion procedure for acoustic waves, Geophysics 44 (1979), $1077-1085$.

[4] R. Courant and D. Hilbert, Methods of Mathematical Physics, vol. II, Wiley-Interscience, New York, 1962.

[5] A. Denisjuk, Integral geometry on the family of semi-spheres, Fractional Calculus and Applied Analysis, 2 1999, 31-46.

[6] J. Fawcett, Inversion of N-dimensional spherical averages, SIAM J. Appl. Math. 45 (1985), 336-341.

[7] A. Faridani, E. L. Ritman and K.T. Smith, Local tomography, SIAM J. Appl. Math., 52 (1992), 459-484.

[8] V. Guillemin, Some remarks on integral geometry, Tech. report, MIT, 1975.

[9] _ On some results of Gelfand in integral geometry, Proceedings Symposia Pure Math. 43 (1985), $149-155$.

[10] V. Guillemin and S. Sternberg, Geometric Asymptotics, American Mathematical Society, Providence, RI, 1977.

[11] M. Haltmeier, T. Schuster, and O. Scherzer, Filtered backprojection for thermoacoustic computed tomography in spherical geometry, Math. Meth. Appl. Sci., 28 (2005), pp. 1919-1937.

[12] L. Hörmander, Fourier Integral Operators, I, Acta Mathematica 127 (1971), 79-183.

[13] J. Klein, Rekonstruktionsverfahren für SAR: Inversion sphärischer Durchschnitte, PhD thesis, Westfälische Wilhelms-Universität, Institut für Numerische und Instrumentelle Mathematik, Münster, Germany, 2003.

[14] P. Kuchment and L. Kunyansky, Mathematics of thermoacoustic tomography, European J. Appl. Math., 19 (2008), 191-224.

[15] A.K. Louis, Approximate inverse for linear and some nonlinear problems, Inverse Problems 12 (1996), $175-190$.

[16] A.K. Louis and E.T. Quinto, Local Tomographic Methods in SONAR, Surveys on Solution Methods for Inverse Problems (Vienna/New York) (D.Colton, H.Engl, A.Louis, J.McLaughlin, and W. Rundell, eds.), Springer Verlag, 2000, pp. 147-154.

[17] S.J. Norton and M. Linzer, Ultrasonic reflectivity imaging in three dimensions: exact inverse scattering solutions for plane, cylindrical, and spherical apertures, IEEE Trans. Biomedical Engineering, 28 (1981), 200-202.

[18] V. Palamodov, Reconstruction from Limited Data of Arc Means, J. Fourier Analysis and Applications 6 (2000), $25-42$.

[19] B. Petersen, Introduction to the Fourier Transform and Pseudo-Differential Operators, Pittman, Boston, 1983.

[20] E.T. Quinto, The dependence of the generalized Radon transform on defining measures, Trans. Amer. Math. Soc. 257 (1980), 331-346.

[21] Support Theorems for the Spherical Radon Transform on Manifolds, International Mathematics Research Notices 2006 (2006), 1-17, Article ID = 67205.

[22] A. Rieder and T. Schuster, The approximate inverse in action II: convergence and stability, Math. Comp. 72 (2003), 1399-1415.

[23] — The approximate inverse in action III: 3D-Doppler tomography, Numer. Math. 97 (2004), 353-378.

[24] T. Schuster, The method of approximate inverse: theory and applications, Lecture Notes in Mathematics 1906, Springer-Verlag, Berlin, 2007.

[25] T. Schuster and E.T. Quinto, On a regularization scheme for linear operators in distribution spaces with an application to the spherical Radon transform, SIAM J. Appl. Math. 65 (2005), no. 4, 1369-1387.

[26] E.M. Stein, Singular Integrals and Differentiability Properties of Functions, Princeton University Press, Princeton New Jersey, 1970.

[27] L.V. Wang, Photoacoustic imaging and Spectroscopy, CRC Press, 2009.

Department of Mathematics, Tufts University, Medford, MA 02155, USA

E-mail address: todd.quinto@tufts.edu

Fakultät für Mathematik, Karlsruher Institut für Technologie (KIT), D-76128 Karlsruhe, GerMANY

E-mail address: andreas.rieder@kit.edu

Fakultät V, Institut für Mathematik, Carl von Ossietzky Universität Oldenburg, 26111 OldenBURG, Germany

E-mail address: thomas.schuster@uni-oldenburg.de 


\section{IWRMM-Preprints seit 2008}

Nr. 08/01 Patrizio Neff, Antje Sydow, Christian Wieners: Numerical approximation of incremental infinitesimal gradient plasticity

Nr. 08/02 Götz Alefeld, Zhengyu Wang: Error Estimation for Nonlinear Complementarity Problems via Linear Systems with Interval Data

Nr. 08/03 Ulrich Kulisch : Complete Interval Arithmetic and its Implementation on the Computer

Nr. 08/04 Armin Lechleiter, Andreas Rieder: Newton Regularizations for Impedance Tomography: Convergence by Local Injectivity

Nr. 08/05 Vu Hoang, Michael Plum, Christian Wieners: A computer-assisted proof for photonic band gaps

Nr. 08/06 Vincent Heuveline, Peter Wittwer: Adaptive boundary conditions for exterior stationary flows in three dimensions

Nr. 08/07 Jan Mayer: Parallel Algorithms for Solving Linear Systems with Sparse Triangular Matrices

Nr. 08/08 Ulrich Kulisch : Begegnungen eines Mathematikers mit Informatik

Nr. 08/09 Tomas Dohnal, Michael Plum, Wolfgang Reichel: Localized Modes of the Linear Periodic Schrödinger Operator with a Nonlocal Perturbation

Nr. 08/10 Götz Alefeld: Verified Numerical Computation for Nonlinear Equations

Nr. 08/11 Götz Alefeld, Zhengyu Wang: Error bounds for complementarity problems with tridiagonal nonlinear functions

Nr. 08/12 Tomas Dohnal, Hannes Uecker: Coupled Mode Equations and Gap Solitons for the 2D Gross-Pitaevskii equation with a non-separable periodic potential

Nr. 09/01 Armin Lechleiter, Andreas Rieder: Towards A General Convergence Theory For Inexact Newton Regularizations

Nr. 09/02 Christian Wieners: A geometric data structure for parallel finite elements and the application to multigrid methods with block smoothing

Nr. 09/03 Arne Schneck: Constrained Hardy Space Approximation

Nr. 09/04 Arne Schneck: Constrained Hardy Space Approximation II: Numerics

Nr. 10/01 Ulrich Kulisch, Van Snyder : The Exact Dot Product As Basic Tool For Long Interval Arithmetic

Nr. 10/02 Tobias Jahnke : An Adaptive Wavelet Method for The Chemical Master Equation

Nr. 10/03 Christof Schütte, Tobias Jahnke : Towards Effective Dynamics in Complex Systems by Markov Kernel Approximation

Nr. 10/04 Tobias Jahnke, Tudor Udrescu : Solving chemical master equations by adaptive wavelet compression

Nr. 10/05 Christian Wieners, Barbara Wohlmuth : A Primal-Dual Finite Element Approximation For A Nonlocal Model in Plasticity

Nr. 10/06 Markus Bürg, Willy Dörfler: Convergence of an adaptive hp finite element strategy in higher space-dimensions

Nr. 10/07 Eric Todd Quinto, Andreas Rieder, Thomas Schuster: Local Inversion of the Sonar Transform Regularized by the Approximate Inverse

Eine aktuelle Liste aller IWRMM-Preprints finden Sie auf: 


\section{Kontakt}

Karlsruher Institut für Technologie (KIT) Institut für Wissenschaftliches Rechnen und Mathematische Modellbildung

Prof. Dr. Christian Wieners Geschäftsführender Direktor

Campus Süd

Engesserstr. 6

76131 Karlsruhe

E-Mail: iwrmm-sekretariat@math.uka.de

www.math. kit.edu/iwrmm/

\section{Herausgeber}

Karlsruher Institut für Technologie (KIT) Kaiserstraße 12 | 76131 Karlsruhe

September 2010 